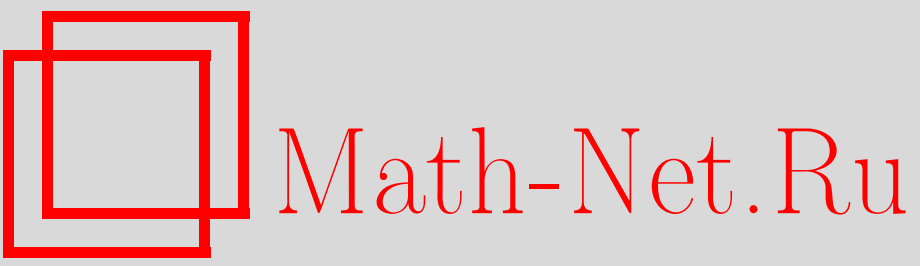

В. В. Сенатов, О неулучшаемых оценках для асимптотических разложений в центральной предельной теореме, Теория вероятн. и ее примен., 2012, том 57, выпуск 4, 649-666

DOI: https://doi.org/10.4213/tvp4472

Использование Общероссийского математического портала Math-Net.Ru подразумевает, что вы прочитали и согласны с пользовательским соглашением

http://www.mathnet.ru/rus/agreement

Параметры загрузки:

IP : 18.209 .158 .208

26 апреля 2023 г., 17:13:34

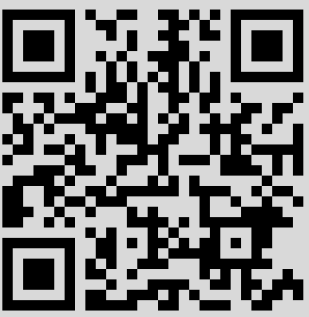




\title{
О НЕУЛУЧШАЕМЫХ ОЩЕНКАХ ДЛЯ АСИМПТОТИЧЕСКИХ РАЗЛОЖЕНИЙ В ЦЕНТРАЛЬНОЙ ПРЕДЕЛЬНОЙ ТЕОРЕМЕ
}

\begin{abstract}
Получены новые асимптотические разложения в центральной предельной теореме с явными оценками точности аппроксимации, которую они гарантируют. Для некоторых из этих оценок справедливо утверждение об их неулучшаемости.
\end{abstract}

Ключевые слова и фразы: центральная предельная теорема, асимптотические разложения, точность аппроксимации.

Цель данной работы - продемонстрировать новые асимптотические разложения в локальной форме центральной предельной теоремы (ЦПТ) для плотностей. Оценки остаточных частей этих разложений выражаются в явном виде. Некоторые из этих оценок являются неулучшаемыми в смысле, который указан ниже. Полученные разложения для плотностей можно преобразовать по простым правилам в асимптотические разложения в интегральной форме ЦПТ (для функций распределения, удовлетворяющих достаточно слабым ограничениям на их гладкость) и в разложения в локальной форме ЦПТ для решетчатых распределений (см. $[1, \S 15$ гл. 4 и 11 гл. 5]).

Пусть $X_{1}, X_{2}, \ldots$ - независимые одинаково распределенные случайные величины с нулевым средним и единичной дисперсией, для которых $\mathbf{E}\left|X_{1}\right|^{m+2}<\infty$, где $m \geqslant 2$ - натуральное число. Пусть $P$ - общее распределение этих случайных величин, $P_{n}$ - распределение нормированной суммы $\left(X_{1}+\cdots+X_{n}\right) n^{-1 / 2}, p_{n}$ - плотность $P_{n}$ (если она существует) и $\varphi(x)=e^{-x^{2} / 2} / \sqrt{2 \pi}$ - плотность стандартного нормального закона. Предположим, что для некоторого числа $\nu>0$

$$
\int_{-\infty}^{\infty}|f(t)|^{\nu} d t<\infty
$$

где $f(t)$ - характеристическая функция распределения $P$. Выполнение этого условия гарантирует существование плотности $p_{n}$ при всех $n \geqslant \nu$

*Московский государственный университет им. М. В. Ломоносова, механико-математический факультет, Москва, Россия; e-mail: v.senatov@yandex.ru 
и справедливость ЦПТ в локальной форме для плотностей, т.е. справедливость утверждения $p_{n}(x) \rightarrow \varphi(x), n \rightarrow \infty$, для всех действительных $x$. Мы хотим указать для $p_{n}(x)$ аппроксимации (они и называются асимптотическими разложениями), позволяющие вычислять $p_{n}(x)$ с точностью $O\left(1 / n^{m / 2}\right), n \rightarrow \infty$, причем нас интересуют те оценки величин $O\left(1 / n^{m / 2}\right)$, которые можно доводить до численных значений.

Обозначим $\alpha_{k}=\mathbf{E} X_{1}^{k}, \beta_{k}=\mathbf{E}\left|X_{1}\right|^{k}, k=1, \ldots, m+2, \alpha_{0}=\beta_{0}=1$ моменты и абсолютные моменты исходных случайных величин. При построении асимптотических разложений используются многочлены Чебышёва-Эрмита $H_{k}(x)=(-1)^{k} \varphi^{(k)}(x) / \varphi(x), k=0,1, \ldots$; первые пять многочленов Чебышёва-Эрмита суть $H_{0}(x) \equiv 1, H_{1}(x)=x, H_{2}(x)=$ $x^{2}-1, H_{3}(x)=x^{3}-3 x, H_{4}(x)=x^{4}-6 x^{2}+3$.

Все известные асимптотические разложения в ЦПТ являются линейными комбинациями функций $H_{k}(x) \varphi(x)$ с коэффициентами, зависящими от моментных характеристик распределения $P$ и числа $n$. Эти моментные характеристики суть комбинации (которые становятся тем сложнее, чем больше число $m$ ) либо моментов, либо семиинвариантов, либо моментов Чебышёва-Эрмита распределения $P$. Далее рассматриваются только разложения, в которых используются моменты Чебышёва-Эрмита. Мы называем моментами Чебышёва-Эрмита числа

$$
\theta_{l}=\theta_{l}(P)=\int_{-\infty}^{\infty} H_{l}(x) P(d x), \quad l=0,1, \ldots, m+2 .
$$

Легко проверить, что $\theta_{0}=1$ для любого распределения $P$, а для распределений с нулевым средним и единичной дисперсией $\theta_{1}=\theta_{2}=0$, $\theta_{3}=\alpha_{3}, \theta_{4}=\alpha_{4}-3$. Моменты Чебышёва-Эрмита достаточно просто вычисляются через моменты распределения $P$, а именно,

$$
\frac{\theta_{l}}{l !}=\sum_{j=0}^{[l / 2]} \frac{\alpha_{l-2 j}}{(l-2 j) !} \frac{(-1)^{j}}{2^{j} j !} .
$$

Мы будем использовать также величины $\theta_{l}^{(r)}$, где $0<r<l$, которые вычисляются по указанной формуле, но в сумме по $j$ слагаемые с $l-2 j>$ $r$, т.е. с $j<(l-r) / 2$, опускаются. В оценках точности аппроксимации, которую гарантируют асимптотические разложения, нам понадобятся величины $\left\|\theta_{l}\right\|$ и $\left\|\theta_{l}^{(r)}\right\|$, которые вычисляются по тем же формулам, что $\theta_{l}$ и $\theta_{l}^{(r)}$, с той разницей, что $(-1)^{j}$ заменяется на 1 и, кроме того, при нечетных $l$ вместо моментов используются их абсолютные значения и вместо старшего момента $\alpha_{l}$ используется $\beta_{l}$.

Известно несколько способов упорядочения слагаемых в асимптотических разложениях. В одном из них слагаемые упорядочиваются по степеням переменной $1 / \sqrt{n}$ (так получаются разложения ЭджвортаКрамера), в другом — по порядкам многочленов Чебышёва-Эрмита (так 
получаются разложения типа Грама-Шарлье). Во избежание недоразумений отметим, что разложения Грама-Шарлье отличаются от разложений Эджворта-Крамера не только перегруппировкой слагаемых. В разложениях Грама-Шарлье коэффициенты при некоторых функциях $H_{k}(x) \varphi(x)$ являются суммами величин, в которые входят множителями биномиальные коэффициенты $C_{n}^{s}$, т.е. многочлены порядка $s$ от переменной $n$. Такие величины суть суммы слагаемых (одночленов), убывающих при росте $n$ с различными скоростями, в том числе и быстрее $1 / n^{m / 2}$. Для того чтобы из разложений Грама-Шарлье получить разложения Эджворта-Крамера, такие одночлены следует перенести в остаточную часть разложения и лишь затем переупорядочить слагаемые в главной части разложения (подробнее см. в $[1$, гл. 4]).

Можно также упорядочивать слагаемые в асимптотических разложениях по числу сомножителей $\theta_{k}$ в суммах, являющихся коэффициентами при функциях $H_{l}(x) \varphi(x)$ (см. [2]). В таких разложениях коэффициенты при функциях $H_{l}(x) \varphi(x)$ формируются с помощью чисел

$$
\Theta_{s, l}=\sum_{\substack{t_{1}+\ldots+t_{s}=l \\ 3 \leqslant t_{1}, \ldots, t_{s}}} \frac{\theta_{t_{1}}}{t_{1} !} \cdots \frac{\theta_{t_{s}}}{t_{s} !},
$$

где суммирование проводится по всем наборам целых чисел $t_{1}, \ldots, t_{s}$, удовлетворяющих указанным условиям. Из разложений, которые приводятся в этой работе, можно получать разложения типа Грама-Шарлье и Эджворта-Крамера (аналогично тому, как это делается в [2]). Мы будем рассматривать только последний способ упорядочения слагаемых.

Нам понадобятся величины $\alpha(T)=\max \{|f(t)|: t \geqslant T\}$ и

$$
B_{k, n}=\frac{1}{2 \pi} \int_{-T \sqrt{n}}^{T \sqrt{n}}|t|^{k} \mu^{n-1}\left(\frac{t}{\sqrt{n}}\right) d t
$$

где $k>0, \mu(t)=\max \left\{|f(t)|, e^{-t^{2} / 2}\right\}$, а $T>0$ - параметр, выбор которого находится в нашем распоряжении. Этот параметр присутствует в теореме, формулировка и доказательство которой приведены ниже. Формальные ограничения на величину $T$ в теореме не налагаются, но по существу они появляются при попытках получить явные оценки величин $B_{k, n}$ для конкретных распределений. Отметим, что для любого распределения с конечным третьим моментом при $|t| \leqslant 1 / \beta_{3}$ справедливо неравенство $\mu(t) \leqslant e^{-t^{2} / 3}$, а для распределения с конечным четвертым моментом можно выбрать $T$ (зависящее от распределения $P$ ) так, что $B_{k, n} \rightarrow B_{k}, n \rightarrow \infty$, где $B_{k}$ - абсолютный момент порядка $k$ стандартного нормального закона, деленный на $\sqrt{2 \pi}$. Из условия, наложенного выше на характеристическую функцию $f(t)$, следует, что $\alpha(T)$ строго меньше единицы для любого $T>0$. 
Теорема. При условиях, наложенных выце на распределение $P$, для любого $n \geqslant \max \{\nu, m+1\}$ плотность $p_{n}(x)$ при всех $-\infty<x<\infty$ допускает представление

$$
\begin{aligned}
p_{n}(x)= & \varphi(x) \\
& +C_{n}^{1}\left(\sum_{l=3}^{m+1} \frac{\theta_{l}}{l ! n^{l / 2}} H_{l}(x) \varphi(x)+\frac{\theta_{m+2}^{(m)}}{(m+2) ! n^{(m+2) / 2}} H_{m+2}(x) \varphi(x)\right) \\
& +\sum_{s=2}^{m} C_{n}^{s} \sum_{l=3 s}^{m+2 s} \frac{\Theta_{s, l}}{n^{l / 2}} H_{l}(x) \varphi(x)+R+\sum_{s=1}^{m}\left(r_{s}+R_{s}\right)+K,
\end{aligned}
$$

$2 \partial e$

$$
\begin{aligned}
|R| \leqslant & \frac{\beta_{m+2}}{(m+2) ! n^{m / 2}} B_{m+2, n} \\
\left|r_{1}\right| \leqslant & C_{n}^{1}\left(\frac{\left\|\theta_{m+3}^{(m+1)}\right\|}{(m+3) ! n^{(m+3) / 2}} B_{m+3, n}+\frac{\left\|\theta_{m+4}^{(m)}\right\|}{(m+4) ! n^{(m+4) / 2}} B_{m+4, n}\right), \\
\left|r_{s}\right| \leqslant & C_{n}^{s} \sum_{l=3(s-1)}^{m-1+2(s-1)}\left|\Theta_{s-1, l}\right|\left(\frac{\left\|\theta_{m+2 s-l+1}\right\|}{(m+2 s-l+1) ! n^{(m+2 s+1) / 2}} B_{m+2 s+1, n}\right. \\
& \left.+\frac{|| \theta_{m+2 s-l+2}^{(m+2 s-l)}||}{(m+2 s-l+2) ! n^{(m+2 s+2) / 2}} B_{m+2 s+2}\right), \quad s=2, \ldots, m, \\
\left|R_{1}\right| \leqslant & C_{n}^{2} \frac{\left|\theta_{m+2}^{(m)}\right|}{(m+2) ! n^{(m+5) / 2}}\left(\frac{\left|\theta_{3}\right|}{3 !} B_{m+5, n}+\frac{\beta_{4}+3}{4 ! \sqrt{n}} B_{m+6, n}\right), \\
\left|R_{s}\right| \leqslant & C_{n}^{s+1} \frac{\left|\Theta_{s, m+2 s}\right|}{n^{(m+2 s+3) / 2}}\left(\frac{\left|\theta_{3}\right|}{3 !} B_{m+2 s+3, n}+\frac{\beta_{4}+3}{4 ! \sqrt{n}} B_{m+2 s+4, n}\right), \\
& s=2, \ldots, m,
\end{aligned}
$$

а величина $K$, убьвающая при росте $n$ экспоненииально бьстро, не превосходит сумми слагаемьх, одно из которьх есть $(\sqrt{n} / \pi) \alpha^{n-\nu}(T) \times$ $\int_{T}^{\infty}|f(t)|^{\nu} d t(T-$ произвольное положительное число), второе равно $e^{-T^{2} n / 2} /(\pi T \sqrt{n})$, а остальнье получаются из главной части разложения заменой моментных характеристик распределения $P$ на их абсолютнье значения и заменой функиий $H_{l}(x) \varphi(x), l \geqslant 3$, на величинь

$$
\frac{1}{\pi} \int_{T \sqrt{n}}^{\infty} t^{l} e^{-t^{2} / 2} d t .
$$

Обратим внимание на то, что в главной части разложения используются моменты, порядки которых не превосходят $m+1$. Момент порядка $m+2$ используется только в оценке остаточной части разложения.

Для доказательства теоремы над понадобится лемма, в которой фигурируют величины $R, r_{s}, R_{s}$, аналогичные тем, что участвуют в теореме, однако смысл этих величин в теореме и в лемме различен; это не 
должно привести к недоразумениям. При доказательстве леммы мы, как правило, не будем выписывать выражения для этих величин, а ограничимся лишь их оценками; это также не должно привести к недоразумениям.

Лемма. Если конечен момент порядка $m+2$, то характеристическая функиия $f^{n}(t / \sqrt{n})$ распределения $P_{n}$ для всех $n \geqslant m+1$ и всех действительных $t$ допускает представление

$$
\begin{aligned}
f^{n}\left(\frac{t}{\sqrt{n}}\right)= & e^{-t^{2} / 2}+C_{n}^{1}\left(\sum_{l=3}^{m+1} \frac{\theta_{l}}{l !}\left(\frac{i t}{\sqrt{n}}\right)^{l}+\frac{\theta_{m+2}^{(m)}}{(m+2) !}\left(\frac{i t}{\sqrt{n}}\right)^{m+2}\right) e^{-t^{2} / 2} \\
& +\sum_{s=2}^{m} C_{n}^{s} \sum_{l=3 s}^{m+2 s} \Theta_{s, l}\left(\frac{i t}{\sqrt{n}}\right)^{l} e^{-t^{2} / 2}+R+\sum_{s=1}^{m}\left(r_{s}+R_{s}\right)
\end{aligned}
$$

әде

$$
\begin{aligned}
|R| \leqslant & \frac{\beta_{m+2}}{(m+2) ! n^{m / 2}}|t|^{m+2} \mu^{n-1}\left(\frac{t}{\sqrt{n}}\right), \\
\left|r_{1}\right| \leqslant & \left(\frac{\left\|\theta_{m+3}^{(m+1)}\right\|}{(m+3) !}+\frac{\left\|\theta_{m+4}^{(m)}\right\|}{(m+4) !}\left|\frac{t}{\sqrt{n}}\right|\right) C_{n}^{1}\left|\frac{t}{\sqrt{n}}\right|^{m+3} \mu^{n-1}\left(\frac{t}{\sqrt{n}}\right), \\
\left|r_{s}\right| \leqslant & \sum_{l=3(s-1)}^{m-1+2(s-1)}\left|\Theta_{s-1, l}\right|\left(\frac{\| \theta_{m+2 s-l+1} \mid}{(m+2 s-l+1) !}+\frac{\| \theta_{m+2 s-l+2}^{(m+2 s-l)} \mid}{(m+2 s-l+2) !}\left|\frac{t}{\sqrt{n}}\right|\right) \\
& \times C_{n}^{s}\left|\frac{t}{\sqrt{n}}\right|^{m+2 s+1} \mid \mu^{n-1}\left(\frac{t}{\sqrt{n}}\right), \quad s=2, \ldots, m, \\
\left|R_{1}\right| \leqslant & \frac{\left|\theta_{m+2}^{(m)}\right|}{(m+2) !}\left(\frac{\left|\theta_{3}\right|}{3 !}+\frac{\beta_{4}+3}{4 !}\left|\frac{t}{\sqrt{n}}\right|\right) C_{n}^{2}\left|\frac{t}{\sqrt{n}}\right|^{m+5} \mu^{n-1}\left(\frac{t}{\sqrt{n}}\right), \\
\left|R_{s}\right| \leqslant & \left|\Theta_{s, m+2 s}\right|\left(\frac{\left|\theta_{3}\right|}{3 !}+\frac{\beta_{4}+3}{4 !}\left|\frac{t}{\sqrt{n}}\right|\right) C_{n}^{s+1}\left|\frac{t}{\sqrt{n}}\right|^{m+2 s+3} \mu^{n-1}\left(\frac{t}{\sqrt{n}}\right), \\
& s=2, \ldots, m .
\end{aligned}
$$

Д о к а з а т ль с т в о. При доказательстве леммы нам будет удобно представлять характеристическую функцию $e^{-t^{2} / 2}$ стандартного нормального закона, предельного в ЦПТ, в виде, аналогичном виду допредельных характеристических функций, т.е. записывать функцию $e^{-t^{2} / 2}$ как $g^{n}(t / \sqrt{n})$, где $g(u)=e^{-u^{2} / 2}$. Мы часто будем опускать аргументы у функций $f, g, \mu$ и у других функций, которые нам понадобятся, это не должно привести к недоразумениям, все эти аргументы суть $t / \sqrt{n}$.

Рассмотрим разность $f^{n}(t / \sqrt{n})-g^{n}(t / \sqrt{n})$. Так как для любых комплексных чисел $a$ и $b$ и любого натурального $k$ справедливо равенство

$$
a^{k}-b^{k}=\sum_{j=0}^{k-1} a^{k-j-1} b^{j}(a-b)
$$


TO

$$
f^{n}-g^{n}=\sum_{j=0}^{n-1} f^{n-j-1} g^{j}(f-g)=\psi \sum_{j=0}^{n-1} f^{n-j-1} g^{j+1},
$$

где $\psi=e^{t^{2} /(2 n)} f(t / \sqrt{n})-1$. Слегка модифицируя доказательство леммы 1 из $[1$, гл. 4, § 7], нетрудно убедиться в справедливости представления

$$
\begin{aligned}
\psi= & \sum_{l=3}^{m+1} \frac{\theta_{l}}{l !}\left(\frac{i t}{\sqrt{n}}\right)^{l}+\frac{\theta_{m+2}^{(m)}}{(m+2) !}\left(\frac{i t}{\sqrt{n}}\right)^{m+2}+\gamma e^{t^{2} /(2 n)} \frac{\beta_{m+2}}{(m+2) !}\left(\frac{t}{\sqrt{n}}\right)^{m+2} \\
& +\gamma e^{t^{2} /(2 n)} \frac{\left\|\theta_{m+3}^{(m+1)}\right\|}{(m+3) !}\left(\frac{t}{\sqrt{n}}\right)^{m+3}+\gamma e^{t^{2} /(2 n)} \frac{\left\|\theta_{m+4}^{(m)}\right\|}{(m+4) !}\left(\frac{t}{\sqrt{n}}\right)^{m+4} .
\end{aligned}
$$

Здесь и далее символ $\gamma$ обозначает комплекснозначные измеримые функции, абсолютные величины которых не превосходят единицы. Эти функции могут быть различны даже в пределах одной формулы.

Используя это представления $\psi$, мы видим, что

$$
\begin{aligned}
f^{n}-g^{n}= & \left(\sum_{l=3}^{m+1} \frac{\theta_{l}}{l !}\left(\frac{i t}{\sqrt{n}}\right)^{l}+\frac{\theta_{m+2}^{(m)}}{(m+2) !}\left(\frac{i t}{\sqrt{n}}\right)^{m+2}\right)\left(\sum_{j=0}^{n-1} f^{n-j-1} g^{j+1}\right) \\
& +\gamma \frac{\beta_{m+2}}{(m+2) !}\left(\frac{t}{\sqrt{n}}\right)^{m+2} \sum_{j=0}^{n-1} f^{n-j-1} g^{j}+r_{1},
\end{aligned}
$$

где

$$
\left\|r_{1}\right\| \leqslant\left(\frac{\left\|\theta_{m+3}^{(m+1)}\right\|}{(m+3) !}\left|\frac{t}{\sqrt{n}}\right|^{m+3}+\frac{\left\|\theta_{m+4}^{(m)}\right\|}{(m+4) !}\left|\frac{t}{\sqrt{n}}\right|^{m+4}\right) n \mu^{n-1} .
$$

Обозначив в правой части (2) первый сомножитель в первом слагаемом через $A$ и вновь воспользовавшись равенством (1), мы можем это слагаемое записать в виде

$$
\begin{aligned}
A \sum_{j_{1}=0}^{n-1} f^{n-j_{1}-1} g^{j_{1}+1} & =A \sum_{j_{1}=0}^{n-2}\left(f^{n-j_{1}-1}-g^{n-j_{1}-1}\right) g^{j_{1}+1}+A n g^{n} \\
& =A C_{n}^{1} g^{n}+\sum_{j_{1}=0}^{n-2}\left(\sum_{j_{2}=0}^{n-j_{1}-2} f^{n-j_{1}-j_{2}-2} g^{j_{2}}(f-g)\right) g^{j_{1}+1} \\
& =A C_{n}^{1} g^{n}+A \sum_{\substack{j_{1}+j_{2} \leqslant n-2 \\
0 \leqslant j_{1}, j_{2}}} f^{n-j_{1}-j_{2}-2} g^{j_{1}+j_{2}+1}(f-g) .
\end{aligned}
$$

В последней сумме суммирование проводится по всем наборам неотрицательных целых чисел $j_{1}, j_{2}$, для которых $j_{1}+j_{2} \leqslant n-2$. Первое слагаемое в правой части последнего равенства участвует в формировании главной части разложения разности $f^{n}-g^{n}$, а второе мы можем 
записать в виде

$$
\left(\sum_{t_{1}=3}^{m+1} \frac{\theta_{t_{1}}}{t_{1} !}\left(\frac{i t}{\sqrt{n}}\right)^{t_{1}} \psi\right)\left(\sum_{\substack{j_{1}+j_{2} \leqslant n-2 \\ 0 \leqslant j_{1}, j_{2}}} f^{n-j_{1}-j_{2}-2} g^{j_{1}+j_{2}+2}\right)+R_{1}
$$

где

$$
\left|R_{1}\right| \leqslant \frac{\left|\theta_{m+2}^{(m)}\right|}{(m+2) !}\left|\frac{t}{\sqrt{n}}\right|^{m+2} C_{n}^{2}\left(\frac{\left|\theta_{3}\right|}{3 !}\left|\frac{t}{\sqrt{n}}\right|^{3}+\frac{\beta_{4}+3}{4 !}\left(\frac{t}{\sqrt{n}}\right)^{4}\right) \mu^{n-1}
$$

Здесь мы воспользовались тем, что для любого $1 \leqslant s \leqslant n-1$ число наборов неотрицательных целых чисел $j_{1}, \ldots, j_{s}$ таких, что $j_{1}+\cdots+j_{s} \leqslant$ $n-s$, равно $C_{n}^{s}$, и почти очевидной оценкой

$$
|f-g| \leqslant \frac{\left|\theta_{3}\right|}{3 !}\left|\frac{t}{\sqrt{n}}\right|^{3}+\frac{\beta_{4}+3}{4 !}\left(\frac{t}{\sqrt{n}}\right)^{4} .
$$

Рассмотрим первый сомножитель в первом слагаемом в (3). Из упоминавшейся леммы 1 из [1] следует, что для любого $3 \leqslant t_{1} \leqslant m+1$

$$
\begin{aligned}
\psi= & \sum_{t_{2}=3}^{m+4-t_{1}} \frac{\theta_{t_{2}}}{t_{2} !}\left(\frac{i t}{\sqrt{n}}\right)^{t_{2}}+\gamma e^{t^{2} /(2 n)} \frac{\left\|\theta_{m+4-t_{1}+1}\right\|}{\left(m+4-t_{1}+1\right) !}\left(\frac{t}{\sqrt{n}}\right)^{m+4-t_{1}+1} \\
& +\gamma e^{t^{2} /(2 n)} \frac{\left\|\theta_{m+4-t_{1}+2}^{\left(m+4-t_{1}\right)}\right\|}{\left(m+4-t_{1}+2\right) !}\left(\frac{t}{\sqrt{n}}\right)^{m+4-t_{1}+2}
\end{aligned}
$$

Отметим, что наибольший порядок моментов, участвующих в формировании правой части последнего равенства, есть $m+2$ : момент $\beta_{m+2}$ участвует в формировании величины $\left\|\theta_{m+2}\right\|$, которая присутствует в правой части последнего равенства при $t_{1}=3$; моменты порядка $m+2$ далее нигде появляться не будут. Из указанных представлений функции $\psi$ следует, что первый сомножитель в первом слагаемом в (3) равен

$$
\sum_{t_{1}=3}^{m+1} \frac{\theta_{t_{1}}}{t_{1} !}\left(\frac{i t}{\sqrt{n}}\right)^{t_{1}} \sum_{t_{2}=3}^{m+4-t_{1}} \frac{\theta_{t_{2}}}{t_{2} !}\left(\frac{i t}{\sqrt{n}}\right)^{t_{2}}+\rho
$$

где

$$
|\rho| \leqslant \sum_{t_{1}=3}^{m+1}\left(\frac{\left|\theta_{t_{1}}\right|}{t_{1} !}\left(\frac{\left\|\theta_{m+5-t_{1}}\right\|}{\left(m+5-t_{1}\right) !}\left|\frac{t}{\sqrt{n}}\right|^{m+5}+\frac{\left\|\theta_{m+6-t_{1}}^{\left(m+4-t_{1}\right)}\right\|}{\left(m+6-t_{1}\right) !}\left|\frac{t}{\sqrt{n}}\right|^{m+6}\right)\right) e^{t^{2} /(2 n)}
$$

Нетрудно проверить, что сумму по $t_{1}$ из (4) можно записать в виде

$$
\sum_{t_{1}=3}^{m+1} \sum_{t_{2}=3}^{m+4-t_{1}} \frac{\theta_{t_{1}}}{t_{1} !} \frac{\theta_{t_{2}}}{t_{2} !}\left(\frac{i t}{\sqrt{n}}\right)^{t_{1}+t_{2}}=\sum_{l=6}^{m+4}\left(\sum_{\substack{t_{1}+t_{2}=l \\ 3 \leqslant t_{1}, t_{2}}} \frac{\theta_{t_{1}}}{t_{1} !} \frac{\theta_{t_{2}}}{t_{2} !}\right)\left(\frac{i t}{\sqrt{n}}\right)^{l} .
$$


Из сказанного следует, что первое слагаемое в (3) равно

$$
\left(\sum_{l=6}^{m+4}\left(\sum_{\substack{t_{1}+t_{2}=l \\ 3 \leqslant t_{1}, t_{2}}} \frac{\theta_{t_{1}}}{t_{1} !} \frac{\theta_{t_{2}}}{t_{2} !}\right)\left(\frac{i t}{\sqrt{n}}\right)^{l}\right)\left(\sum_{\substack{j_{1}+j_{2} \leqslant n-2 \\ 0 \leqslant j_{1}, j_{2}}} f^{n-j_{1}-j_{2}-2} g^{j_{1}+j_{2}+2}\right)+r_{2},
$$

где

$$
\left|r_{2}\right| \leqslant \sum_{t_{1}=3}^{m+1}\left(\frac{\left|\theta_{t_{1}}\right|}{t_{1} !}\left(\frac{\left\|\theta_{m+5-t_{1}}\right\|}{\left(m+5-t_{1}\right) !}+\frac{\left\|\theta_{m+6-t_{1}}^{\left(m+4-t_{1}\right)}\right\|}{\left(m+6-t_{1}\right) !}\left|\frac{t}{\sqrt{n}}\right|\right)\right)\left|\frac{t}{\sqrt{n}}\right|^{m+5} C_{n}^{2} \mu^{m-1} .
$$

Второй сомножитель в первом слагаемом (5) можно записать в виде

$$
\begin{aligned}
& \sum_{\substack{j_{1}+j_{2} \leqslant n-3 \\
0 \leqslant j_{1}, j_{2}}}\left(f^{n-j_{1}-j_{2}-2}-g^{n-j_{1}-j_{2}-2}\right) g^{j_{1}+j_{2}+2}+C_{n}^{2} g^{n} \\
& =\sum_{\substack{j_{1}+j_{2} \leqslant n-3 \\
0 \leqslant j_{1}, j_{2}}}\left(\sum_{j_{3}=0}^{n-j_{1}-j_{2}-3} f^{n-j_{1}-j_{2}-j_{3}-3} g^{j_{3}}(f-g)\right) g^{j_{1}+j_{2}+2}+C_{n}^{2} g^{n} \\
& =C_{n}^{2} g^{n}+\sum_{\substack{j_{1}+j_{2}+j_{3} \leqslant n-3 \\
0 \leqslant j_{1}, j_{2}, j_{3}}} f^{n-j_{1}-j_{2}-j_{3}-3} g^{j_{1}+j_{2}+j_{3}+2}(f-g) .
\end{aligned}
$$

Поэтому первое слагаемое в (5) равно

$$
\begin{aligned}
C_{n}^{2} & \sum_{l=6}^{m+4}\left(\sum_{\substack{t_{1}+t_{2}=l \\
3 \leqslant t_{1}, t_{2}}} \frac{\theta_{t_{1}}}{t_{1} !} \frac{\theta_{t_{2}}}{t_{2} !}\right)\left(\frac{i t}{\sqrt{n}}\right)^{l} g^{n} \\
& +\left(\sum_{\substack{l=6 \\
m+3}}\left(\sum_{\substack{t_{1}+t_{2}=l \\
3 \leqslant t_{1}, t_{2}}} \frac{\theta_{t_{1}}}{t_{1} !} \frac{\theta_{t_{2}}}{t_{2} !}\right)\left(\frac{i t}{\sqrt{n}}\right)^{l} \psi\right) \\
& \times\left(\sum_{\substack{j_{1}+j_{2}+j_{3} \leqslant n-3 \\
0 \leqslant j_{1}, j_{2}, j_{3}}} f^{n-j_{1}-j_{2}-j_{3}-3} g^{j_{1}+j_{2}+j_{3}+3}\right)+R_{2},
\end{aligned}
$$

где

$$
\left|R_{2}\right| \leqslant\left|\sum_{\substack{t_{1}+t_{2}=m+4 \\ 3 \leqslant t_{1}, t_{2}}} \frac{\theta_{t_{1}}}{t_{1} !} \frac{\theta_{t_{2}}}{t_{2} !}\right|\left|\frac{t}{\sqrt{n}}\right|^{m+4} C_{n}^{3}\left(\frac{\left|\theta_{3}\right|}{3 !}\left|\frac{t}{\sqrt{n}}\right|^{3}+\frac{\beta_{4}+3}{4 !}\left(\frac{t}{\sqrt{n}}\right)^{4}\right) \mu^{n-1} .
$$

При $m=2$ сумма по $6 \leqslant l \leqslant m+4$ состоит из единственного слагаемого с $l=6$, сумма по $t_{1}+t_{2}=6$ состоит из единственного слагаемого $\left(\theta_{3} / 3 !\right)^{2} ;$ предпоследнее слагаемое в (6) следует опустить, и доказательство леммы на этом заканчивается. 
Далее мы считаем, что $m \geqslant 3$. Положим

$$
\begin{aligned}
U_{s} & =\sum_{V_{s+1}}=\sum_{\substack{l=3 s \\
j_{1}+\cdots+j_{s+1} \leqslant n-(s+1) \\
0 \leqslant j_{1}, \ldots, j_{s+1}}} f^{n-1+2 s} \Theta_{s, l}\left(\frac{i t}{\sqrt{n}}\right)^{l} \psi, \\
j_{1}-\cdots-j_{s+1}-(s+1) & g^{j_{1}+\cdots+j_{s+1}+s+1}
\end{aligned}
$$

и $W_{s}=U_{s} V_{s+1}$, где $s=1, \ldots, m-1$.

Легко проверить, что $W_{1}$ совпадает с первым слагаемым в (3), а $W_{2}$ - с предпоследним слагаемым в $(6)$, и из сказанного выше следует, что

$$
W_{1}=C_{n}^{2} \sum_{l=6}^{m+4} \Theta_{2, l}\left(\frac{i t}{\sqrt{n}}\right)^{l} g^{n}+W_{2}+r_{2}+R_{2} .
$$

Оказывается, что для любого $1 \leqslant s \leqslant m-2$

$$
W_{s}=C_{n}^{s+1} \sum_{l=3(s+1)}^{m+2(s+1)} \Theta_{s+1, l}\left(\frac{i t}{\sqrt{n}}\right)^{l} g^{n}+W_{s+1}+r_{s+1}+R_{s+1},
$$

способ формирования величин $r_{s+1}$ и $R_{s+1}$ станет ясен из дальнейшего, а их оценки будут указаны ниже.

Действительно, для каждого $l$ из области суммирования в выражении для $U_{s}$ мы можем представить величину $\psi$ в виде

$$
\begin{aligned}
\psi= & \sum_{t_{s+1}=3}^{m+2(s+1)-l} \frac{\theta_{t_{s+1}}}{t_{s+1} !}\left(\frac{i t}{\sqrt{n}}\right)^{t_{s+1}} \\
& +\gamma e^{t^{2} /(2 n)} \frac{\left\|\theta_{m+2(s+1)-l+1}\right\|}{(m+2(s+1)-l+1) !}\left(\frac{t}{\sqrt{n}}\right)^{m+2(s+1)-l+1} \\
& +\gamma e^{t^{2} /(2 n)} \frac{\left\|\theta_{m+2(s+1)-l+2}^{(m+2(s+1)}\right\|}{(m+2(s+1)-l+2) !}\left(\frac{t}{\sqrt{n}}\right)^{m+2(s+1)-l+2} .
\end{aligned}
$$

Отметим, что при $2 \leqslant s$ суммирование в первой сумме ведется по непустому множеству индексов и в правой части последнего равенства используются моменты порядков не выше $m+3-s \leqslant m+1$. Из этих представлений функции $\psi$ следует, что

$$
W_{s}=\left(\sum_{l=3 s}^{m-1+2 s} \Theta_{s, l}\left(\frac{i t}{\sqrt{n}}\right)^{l} \sum_{t_{s+1}=3}^{m+2(s+1)-l} \frac{\theta_{t_{s+1}}}{t_{s+1} !}\left(\frac{i t}{\sqrt{n}}\right)^{t_{s+1}}\right) V_{s+1}+r_{s+1},
$$

где

$$
\begin{aligned}
\left|r_{s+1}\right| \leqslant \sum_{l=3 s}^{m-1+2 s}\left|\Theta_{s, l}\right| & \left(\frac{\left\|\theta_{m+2(s+1)-l+1}\right\|}{(m+2(s+1)-l+1) !}\right. \\
& \left.+\frac{\left\|\theta_{m+2(s+1)-l+2}^{(m+2(s+1)-l)}\right\|}{(m+2(s+1)-l+2) !}\left|\frac{t}{\sqrt{n}}\right|\right)\left|\frac{t}{\sqrt{n}}\right|^{m+2(s+1)+1} C_{n}^{s+1} \mu^{n-1} .
\end{aligned}
$$


Первый сомножитель в первом слагаемом в правой части (8) можно записать в виде

$$
\begin{aligned}
& \sum_{l=3 s}^{m-1+2 s}\left(\sum_{\substack{t_{1}+\ldots+t_{s}=l \\
3 \leqslant t_{1}, \ldots, t_{s}}} \frac{\theta_{t_{1}}}{t_{1} !} \cdots \frac{\theta_{t_{s}}}{t_{s} !}\left(\frac{i t}{\sqrt{n}}\right)^{t_{1}+\cdots+t_{s}}\right. \\
& \left.\times \sum_{t_{s+1}=3}^{m+2(s+1)-\left(t_{1}+\cdots+t_{s}\right)} \frac{\theta_{t_{s+1}}}{t_{s+1} !}\left(\frac{i t}{\sqrt{n}}\right)^{t_{s+1}}\right) \\
& =\sum_{l=3 s}^{m-1+2 s} \sum_{\substack{t_{1}+\cdots,+t_{s}=l \\
3 \leqslant t_{1}, \ldots, t_{s}}}^{m+2(s+1)-\left(t_{1}+\cdots+t_{s}\right)} \frac{\theta_{t_{1}}}{t_{1} !} \cdots \frac{\theta_{t_{s+1}}}{t_{s+1} !}\left(\frac{i t}{\sqrt{n}}\right)^{t_{1}+\cdots+t_{s+1}} \\
& =\sum_{l=3(s+1)}^{m+2(s+1)} \Theta_{s+1, l}\left(\frac{i t}{\sqrt{n}}\right)^{l} \text {. }
\end{aligned}
$$

Второй сомножитель в первом слагаемом в правой части (8) можно преобразовать следующим образом:

$$
\begin{aligned}
& V_{s+1}=\sum_{\substack{j_{1}+\cdots+j_{s+1} \leqslant n-(s+1)-1 \\
0 \leqslant j_{1}, \ldots, j_{s+1}}}\left(f^{n-j_{1}-\cdots-j_{s+1}-(s+1)}\right. \\
& \left.-g^{n-j_{1}-\cdots-j_{s+1}-(s+1)}\right) g^{j_{1}+\cdots+j_{s+1}+s+1}+C_{n}^{s+1} g^{n} \\
& =C_{n}^{s+1} g^{n}+\sum_{\substack{j_{1}+\cdots+j_{s+1} \leqslant n-(s+1)-1 \\
0 \leqslant j_{1}, \ldots, j_{s+1}}} \sum_{j_{s+2}=0}^{n-j_{1}-\cdots-j_{s+1}-(s+1)-1} f^{n-j_{1}-\cdots-j_{s+2}-(s+2)} \\
& \times g^{j_{1}+\cdots+j_{s+2}+s+1}(f-g) \\
& =C_{n}^{s+1} g^{n} \sum_{\substack{j_{1}+\cdots+j_{s+2} \leqslant n-(s+2) \\
0 \leqslant j_{1}, \ldots, j_{s+2}}} f^{n-j_{1}-\cdots-j_{s+2}-(s+2)} g^{j_{1}+\cdots+j_{s+2}+s+1}(f-g) .
\end{aligned}
$$

Произведение правой части (9) и первого слагаемого из правой части (10) присутствует в (7), оно участвует в формировании главной части разложения $f^{n}-g^{n}$. Произведение суммы из правой части (9) без последнего слагаемого и суммы по $j_{1}, \ldots, j_{s+2}$ из правой части $(10)$, где мы можем записать $f-g$ как $g \psi$, равно произведению $U_{s+1} V_{s+2}$, т.е. величине $W_{s+1}$. Наконец, произведение последнего слагаемого из (9) и суммы по $j_{1}, \ldots, j_{s+2}$ из $(10)$ дает величину $R_{s+1}$,

$$
\left|R_{s+1}\right| \leqslant\left|\Theta_{s+1, m+2(s+1)}\right|\left|\frac{t}{\sqrt{n}}\right|^{m+2(s+1)} C_{n}^{s+2} \mu^{n-1}\left(\frac{\left|\theta_{3}\right|}{3 !}\left|\frac{t}{\sqrt{n}}\right|^{3}+\frac{\beta_{4}+3}{4 !}\left(\frac{t}{\sqrt{n}}\right)^{4}\right) .
$$

Теперь равенство (7) становится очевидным. 
Используя равенство (7) при $s=1, \ldots, m-2$, мы видим, что

$$
\begin{aligned}
f^{n}-g^{n}= & C_{n}^{1}\left(\sum_{l=3}^{m+1} \frac{\theta_{l}}{l !}\left(\frac{i t}{\sqrt{n}}\right)^{l}+\frac{\theta_{m+2}^{(m)}}{(m+2) !}\left(\frac{i t}{\sqrt{n}}\right)^{m+2}\right) g^{n} \\
& +\sum_{s=2}^{m-1} C_{n}^{s} \sum_{l=3 s}^{m+2 s} \Theta_{s, l}\left(\frac{i t}{\sqrt{n}}\right)^{l} g^{n}+W_{m-1}+\sum_{s=1}^{m-1}\left(r_{s}+R_{s}\right) .
\end{aligned}
$$

Рассмотрим величину $W_{m-1}$. Так как $U_{m-1}$ состоит из единственного слагаемого, то

$$
W_{m-1}=\Theta_{m-1,3(m-1)}\left(\frac{i t}{\sqrt{n}}\right)^{3(m-1)} \psi \sum_{\substack{j_{1}+\cdots+j_{m} \leqslant n-m \\ 0 \leqslant j_{1}, \ldots, j_{m}}} f^{n-j_{1}-\cdots-j_{m}-m} g^{j_{1}+\cdots+j_{m}+m} .
$$

Для величины $\psi$ справедливо представление

$$
\psi=\frac{\theta_{3}}{3 !}\left(\frac{i t}{\sqrt{n}}\right)^{3}+\gamma e^{t^{2} /(2 n)} \frac{\left\|\theta_{4}\right\|}{4 !}\left(\frac{t}{\sqrt{n}}\right)^{4}+\gamma e^{t^{2} /(2 n)} \frac{\left\|\theta_{5}^{(3)}\right\|}{5 !}\left(\frac{t}{\sqrt{n}}\right)^{5},
$$

a

$$
\Theta_{m-1,3(m-1)}=\sum_{\substack{t_{1}+\cdots+t_{m-1}=3(m-1) \\ 3 \leqslant t_{1}, \ldots, t_{m-1}}} \frac{\theta_{t_{1}}}{t_{1} !} \cdots \frac{\theta_{t_{m-1}}}{t_{m-1} !}=\left(\frac{\theta_{3}}{3 !}\right)^{m-1} .
$$

Поэтому

$$
W_{m-1}=\Theta_{m, 3 m}\left(\frac{i t}{\sqrt{n}}\right)^{3 m} \sum_{\substack{j_{1}+\ldots+j_{m} \leqslant n-m \\ 0 \leqslant j_{1}, \ldots, j_{m}}} f^{n-j_{1}-\cdots-j_{m}-m} g^{j_{1}+\cdots+j_{m}+m}+r_{m},
$$

где

$$
\left|r_{m}\right| \leqslant\left|\frac{\theta_{3}}{3 !}\right|^{m-1}\left(\frac{\left\|\theta_{4}\right\|}{4 !}+\frac{\left\|\theta_{5}^{(3)}\right\|}{5 !}\left|\frac{t}{\sqrt{n}}\right|\right)\left|\frac{t}{\sqrt{n}}\right|^{3 m+1} C_{n}^{m} \mu^{n-1}
$$

Правая часть (11) без величин $r_{m}$ равна

$$
\begin{aligned}
\Theta_{m, 3 m}\left(\frac{i t}{\sqrt{n}}\right)^{3 m} & \left(C_{n}^{m} g^{n}\right. \\
& \left.+\sum_{\substack{j_{1}+\cdots+j_{m+1} \leqslant n-(m+1) \\
0 \leqslant j_{1}, \ldots, j_{m}+1}} f^{n-j_{1}-\cdots-j_{m+1}-(m+1)} g^{j_{1}+\cdots+j_{m+1}+m+1}\right) \\
=\Theta_{m, 3 m} C_{n}^{m} g^{n} & +R_{m},
\end{aligned}
$$

где

$$
\left|R_{m}\right| \leqslant\left|\Theta_{m, 3 m}\right|\left|\frac{t}{\sqrt{n}}\right|^{3 m+3}\left(\frac{\left|\theta_{3}\right|}{3 !}+\frac{\beta_{4}+3}{4 !}\left|\frac{t}{\sqrt{n}}\right|\right) C_{n}^{m+1} \mu^{n-1} .
$$

Лемма доказана. 
Д ок а з а т ель с т в о т е о р е м ы основано на том, что при $n \geqslant \nu$ мы можем разность $p_{n}(x)-\varphi(x)$ вычислять с помощью формулы обращения

$$
\begin{aligned}
p_{n}(x)-\varphi(x)= & \frac{1}{2 \pi} \int_{-\infty}^{\infty} e^{-i t x}\left(f^{n}\left(\frac{t}{\sqrt{n}}\right)-e^{-t^{2} / 2}\right) d t \\
= & \frac{1}{2 \pi} \int_{-T \sqrt{n}}^{T \sqrt{n}} e^{-i t x}\left(f^{n}\left(\frac{t}{\sqrt{n}}\right)-e^{-t^{2} / 2}\right) d t \\
& +\frac{1}{2 \pi} \int_{|t|>T \sqrt{n}} e^{-i t x} f^{n}\left(\frac{t}{\sqrt{n}}\right) d t-\frac{1}{2 \pi} \int_{|t|>T \sqrt{n}} e^{-i t x} e^{-t^{2} / 2} d t
\end{aligned}
$$

Появление свободного параметра $T$ связано с тем, что для некоторых распределений $P$ содержательные оценки функции $\mu(t)$ можно получать лишь для конечных интервалов $-T \leqslant t \leqslant T$.

Абсолютная величина последнего слагаемого в (12) не превосходит

$$
\frac{1}{\pi} \int_{T \sqrt{n}}^{\infty} e^{-t^{2} / 2} d t<\frac{e^{-T^{2} n / 2}}{\pi T \sqrt{n}}
$$

а абсолютная величина предпоследнего слагаемого не превосходит

$$
\frac{1}{\pi} \int_{T \sqrt{n}}^{\infty}\left|f\left(\frac{t}{\sqrt{n}}\right)\right|^{n} d t=\frac{\sqrt{n}}{\pi} \int_{T}^{\infty}|f(t)|^{n} d t \leqslant \frac{\sqrt{n}}{\pi} \alpha^{n-\nu}(T) \int_{T}^{\infty}|f(t)|^{\nu} d t .
$$

При вычислении первого слагаемого в правой части (12) мы можем воспользоваться утверждением леммы. Оценки слагаемых в остаточной части разложения разности $f^{n}(t / \sqrt{n}) e^{-t^{2} / 2}$, т.е. оценки слагаемых, содержащих функции $|t|^{k} \mu^{n-1}(t / \sqrt{n})$, проводятся без труда. При интегрировании слагаемых главной части разложения указанной разности нужно лишь учесть, что

$$
\begin{aligned}
\frac{1}{2 \pi} \int_{-T \sqrt{n}}^{T \sqrt{n}} e^{-i t x}(i t)^{l} e^{-t^{2} / 2} d t & =\frac{1}{2 \pi} \int_{-\infty}^{\infty} e^{-i t x}(i t)^{l} e^{-t^{2} / 2} d t+k \\
& =H_{l}(x) \varphi(x)+k,
\end{aligned}
$$

где

$$
|k| \leqslant \frac{1}{\pi} \int_{T \sqrt{n}}^{\infty} t^{l} e^{-t^{2} / 2} d t .
$$

После этих пояснений утверждение теоремы становится очевидным. Приведем три следствия теоремы.

Для распределений с конечным четвертым моментом $п р и ~ n \geqslant$ $\max (\nu, 3)$

$$
\begin{aligned}
p_{n}(x)= & \varphi(x)+\frac{\theta_{3}}{3 ! \sqrt{n}} H_{3}(x) \varphi(x) \\
& +\frac{1}{n}\left(\frac{\theta_{4}^{(2)}}{4 !} H_{4}(x)+\frac{n-1}{2 n}\left(\frac{\theta_{3}}{3 !}\right)^{2} H_{6}(x)\right) \varphi(x)+R+K,
\end{aligned}
$$


где

$$
\begin{aligned}
|R| \leqslant & \frac{\beta_{4}}{4 !} \frac{B_{4, n}}{n}+\frac{\left\|\theta_{5}^{(3)}\right\|}{5 !} \frac{B_{5, n}}{n^{3 / 2}}+\left(\frac{n-1}{2 n} \frac{\left|\theta_{3}\right|}{3 !} \frac{\left\|\theta_{4}\right\|}{4 !}+\frac{n-1}{2 n} \frac{\left|\theta_{3}\right|}{3 !} \frac{\left|\theta_{4}^{(2)}\right|}{4 !}\right) \frac{B_{7, n}}{n^{3 / 2}} \\
& +\frac{(n-1)(n-2)}{3 ! n^{2}}\left(\frac{\theta_{3}}{3 !}\right)^{3} \frac{B_{9, n}}{n^{3 / 2}} \\
& +\frac{\left\|\theta_{6}^{(2)}\right\|}{6 !} \frac{B_{6, n}}{n^{2}}+\frac{n-1}{2 n}\left(\frac{\left|\theta_{3}\right|}{3 !} \frac{\left\|\theta_{5}^{(3)}\right\|}{5 !} \frac{\left|\theta_{4}^{(2)}\right|}{4 !} \frac{\beta_{4}+3}{4 !}\right) \frac{B_{8, n}}{n^{2}} \\
& +\frac{(n-1)(n-2)}{6 n^{2}}\left(\frac{\theta_{3}}{3 !}\right)^{2} \frac{\beta_{4}+3}{4 !} \frac{B_{10, n}}{n^{2}} .
\end{aligned}
$$

Для распределений с конечным пятым моментом при $n \geqslant \max (\nu, 4)$

$$
\begin{aligned}
p_{n}(x)= & \varphi(x)+\frac{\theta_{3}}{3 ! \sqrt{n}} H_{3}(x) \varphi(x)+\frac{1}{n}\left(\frac{\theta_{4}}{4 !} H_{4}(x)+\frac{n-1}{2 n}\left(\frac{\theta_{3}}{3 !}\right)^{2} H_{6}(x)\right) \varphi(x) \\
& +\frac{1}{n^{3 / 2}}\left(\frac{\theta_{5}^{(3)}}{5 !} H_{5}(x)+\frac{n-1}{n} \frac{\theta_{3}}{3 !} \frac{\theta_{4}}{4 !} H_{7}(x)\right. \\
& \left.+\frac{(n-1)(n-2)}{6 n^{2}}\left(\frac{\theta_{3}}{3 !}\right)^{3} H_{9}(x)\right) \varphi(x)+R+K
\end{aligned}
$$

$2 \partial e$

$$
\begin{aligned}
|R| \leqslant & \frac{\beta_{5}}{5 !} \frac{B_{5, n}}{n^{3 / 2}}+\frac{\left\|\theta_{6}^{(4)}\right\|}{6 !} \frac{B_{6, n}}{n^{2}} \\
& +\frac{n-1}{2 n}\left(\frac{\left|\theta_{3}\right|}{3 !} \frac{\left\|\theta_{5}\right\|}{5 !}+\frac{\left|\theta_{4}\right|}{4 !} \frac{\left\|\theta_{4}\right\|}{4 !}+\frac{\left|\theta_{3}\right|}{3 !} \frac{\left|\theta_{5}^{(3)}\right|}{5 !}\right) \frac{B_{8, n}}{n^{2}} \\
& +\frac{(n-1)(n-2)}{6 n^{2}}\left(\left(\frac{\theta_{3}}{3 !}\right)^{2} \frac{\left\|\theta_{4}\right\|}{4 !}+2\left(\frac{\theta_{3}}{3 !}\right)^{2} \frac{\left|\theta_{4}\right|}{4 !}\right) \frac{B_{10, n}}{n^{2}} \\
& +\frac{(n-1)(n-2)(n-3)}{24 n^{3}}\left(\frac{\theta_{3}}{3 !}\right)^{4} \frac{B_{12, n}}{n^{2}} \\
& +\frac{\left\|\theta_{7}^{(3)}\right\|}{7 !} \frac{B_{7, n}}{n^{5 / 2}}+\frac{1}{2}\left(\frac{\left|\theta_{3}\right|}{3 !} \frac{\left\|\theta_{6}^{(4)}\right\|}{6 !}+\frac{\left|\theta_{4}\right|}{4 !} \frac{\left\|\theta_{5}^{(3)}\right\|}{5 !}+\frac{\beta_{4}+3}{4 !} \frac{\left|\theta_{5}^{(3)}\right|}{5 !}\right) \frac{B_{9, n}}{n^{5 / 2}} \\
& +\frac{1}{6}\left(\left(\frac{\theta_{3}}{3 !}\right)^{2} \frac{\left\|\theta_{5}^{(3)}\right\|}{5 !}+2 \frac{\left|\theta_{3}\right|}{3 !} \frac{\left|\theta_{4}\right|}{4 !} \frac{\beta_{4}+3}{4 !}\right) \frac{B_{11, n}}{n^{5 / 2}}+\frac{1}{4 !}\left|\frac{\theta_{3}}{3 !}\right|^{3} \frac{\beta_{4}+3}{4 !} \frac{B_{13, n}}{n^{5 / 2}} .
\end{aligned}
$$
$\max (\nu, 5)$

$$
\begin{aligned}
p_{n}(x)= & \varphi(x)+\frac{\theta_{3}}{3 ! \sqrt{n}} H_{3}(x) \varphi(x) \\
& +\frac{1}{n}\left(\frac{\theta_{4}}{4 !} H_{4}(x)+\left(\frac{\theta_{6}^{(4)}}{6 ! n}+\frac{n-1}{2 n}\left(\frac{\theta_{3}}{3 !}\right)^{2}\right) H_{6}(x)\right) \varphi(x)
\end{aligned}
$$




$$
\begin{aligned}
& +\frac{1}{n^{3 / 2}}\left(\frac{\theta_{5}}{5 !} H_{5}(x)+\frac{n-1}{n} \frac{\theta_{3}}{3 !} \frac{\theta_{4}}{4 !} H_{7}(x)\right. \\
& \left.\quad+\frac{(n-1)(n-2)}{6 n^{2}}\left(\frac{\theta_{3}}{3 !}\right)^{3} H_{9}(x)\right) \varphi(x) \\
& +\frac{1}{n^{2}}\left(\frac{n-1}{n} \frac{\theta_{3}}{3 !} \frac{\theta_{5}}{5 !}+\frac{n-1}{2 n}\left(\frac{\theta_{4}}{4 !}\right)^{2}\right) H_{8}(x) \varphi(x) \\
& +\frac{1}{n^{2}} \frac{(n-1)(n-2)}{2 n^{2}}\left(\frac{\theta_{3}}{3 !}\right)^{2} \frac{\theta_{4}}{4 !} H_{10}(x) \varphi(x) \\
& +\frac{1}{n^{2}} \frac{(n-1)(n-2)(n-3)}{4 ! n^{3}}\left(\frac{\theta_{3}}{3 !}\right)^{4} H_{12}(x) \varphi(x)+R+K,
\end{aligned}
$$

гдe

$$
\begin{aligned}
& |R| \leqslant \frac{\beta_{6}}{6 !} \frac{B_{6, n}}{n^{2}}+\frac{\left\|\theta_{7}^{(5)}\right\|}{7 !} \frac{B_{7, n}}{n^{5 / 2}} \\
& +\frac{1}{2}\left(\left(\frac{\left|\theta_{3}\right|}{3 !} \frac{\left\|\theta_{6}\right\|}{6 !}+\frac{\left|\theta_{3}\right|}{3 !} \frac{\left|\theta_{6}^{(4)}\right|}{6 !}\right)+\left(\frac{\left|\theta_{4}\right|}{4 !} \frac{\left\|\theta_{5}\right\|}{5 !}+\frac{\left\|\theta_{4}\right\|}{4 !} \frac{\left|\theta_{5}\right|}{5 !}\right)\right) \frac{B_{9, n}}{n^{5 / 2}} \\
& +\frac{1}{6}\left(\left(\frac{\theta_{3}}{3 !}\right)^{2} \frac{\left\|\theta_{5}\right\|}{5 !}+2\left(\frac{\theta_{3}}{3 !}\right)^{2} \frac{\left|\theta_{5}\right|}{5 !}+\frac{\left|\theta_{3}\right|}{3 !}\left(\frac{\theta_{4}}{4 !}\right)^{2}+2 \frac{\left|\theta_{3}\right|}{3 !} \frac{\left|\theta_{4}\right|}{4 !} \frac{\left\|\theta_{4}\right\|}{4 !}\right) \frac{B_{11, n}}{n^{5 / 2}} \\
& +\frac{1}{4 !}\left(\left|\frac{\theta_{3}}{3 !}\right|^{3} \frac{\left\|\theta_{4}\right\|}{4 !}+3\left|\frac{\theta_{3}}{3 !}\right|^{3} \frac{\left|\theta_{4}\right|}{4 !}\right) \frac{B_{13, n}}{n^{5 / 2}}+\frac{1}{5 !}\left|\frac{\theta_{3}}{3 !}\right|^{5} \frac{B_{15, n}}{n^{5 / 2}}+\frac{\left\|\theta_{8}^{(4)}\right\|}{8 !} \frac{B_{8, n}}{n^{3}} \\
& +\frac{1}{2}\left(\frac{\left|\theta_{3}\right|}{3 !} \frac{\left\|\theta_{7}^{(5)}\right\|}{7 !}+\left(\frac{\left|\theta_{4}\right|}{4 !} \frac{\left\|\theta_{6}^{(4)}\right\|}{6 !}+\frac{\beta_{4}+3}{4 !} \frac{\left|\theta_{6}^{(4)}\right|}{6 !}\right)+\frac{\left|\theta_{5}\right|}{5 !} \frac{\left\|\theta_{5}^{(3)}\right\|}{5 !}\right) \frac{B_{10, n}}{n^{3}} \\
& +\frac{1}{6}\left(\left(\frac{\theta_{3}}{3 !}\right)^{2} \frac{\left\|\theta_{6}^{(4)}\right\|}{6 !}+2 \frac{\left|\theta_{3}\right|}{3 !} \frac{\theta_{4}}{4 !} \frac{\left\|\theta_{5}^{(3)}\right\|}{5 !}\right. \\
& \left.+2 \frac{\left|\theta_{3}\right|}{3 !} \frac{\beta_{4}+3}{4 !} \frac{\left|\theta_{5}\right|}{5 !}+\left(\frac{\theta_{4}}{4 !}\right)^{2} \frac{\beta_{4}+3}{4 !}\right) \frac{B_{12, n}}{n^{3}} \\
& +\frac{1}{4 !}\left(\left|\frac{\theta_{3}}{3 !}\right|^{3} \frac{\left\|\theta_{5}^{(3)}\right\|}{5 !}+3\left(\frac{\theta_{3}}{3 !}\right)^{2} \frac{\left|\theta_{4}\right|}{4 !} \frac{\beta_{4}+3}{4 !}\right) \frac{B_{14, n}}{n^{3}} \\
& +\frac{1}{5 !}\left(\frac{\theta_{3}}{3 !}\right)^{4} \frac{\beta_{4}+3}{4 !} \frac{B_{16, n}}{n^{3}} \text {. }
\end{aligned}
$$

О величинах $K$ сказано в формулировке теоремы. Отметим, что $\theta_{4}^{(2)} / 4 !=-1 / 8, \theta_{5}^{(3)} / 5 !=-\alpha_{3} / 12, \theta_{6}^{(2)} / 6 !=1 / 24, \theta_{6}^{(4)} / 6 !=-\alpha_{4} / 48+1 / 24$ и вместо величины $\left\|\theta_{4}\right\| / 4$ ! $\left(=\left(\beta_{4}+9\right) / 4\right.$ !) можно использовать $\left(\beta_{4}+3\right) / 4$ !, вместо величины $\left\|\theta_{6}^{(2)}\right\| / 6$ ! $(=1 / 12)$ можно использовать $1 / 24$, вместо величины $\left\|\theta_{6}^{(4)}\right\| / 6 !\left(=\alpha_{4} / 48+1 / 12\right)$ можно использовать $\alpha_{4} / 48+1 / 24$ и вместо $\left\|\theta_{8}^{(4)}\right\| / 8 !\left(=\alpha_{4} / 192+5 / 384\right)$ можно использовать $\alpha_{4} / 192+1 / 128$.

Способ упорядочения слагаемых в главных частях указанных разложений связан с тем, что в общем случае (это проявляется и в следствиях теоремы) в них присутствует ровно одно слагаемое, убывающее при росте $n$ как $1 / \sqrt{n}$, оно связано с многочленом $H_{3}(x)$; присутствуют два 
слагаемых, убывающих как $1 / n$, они связаны с многочленами $H_{4}(x)$ и $H_{6}(x)$; при $m \geqslant 3$ присутствуют три слагаемых, убывающих как $1 / n^{3 / 2}$, они связаны с многочленами $H_{5}(x), H_{7}(x)$ и $H_{9}(x)$, и т.д.; слагаемые, убывающие как $1 / n^{k / 2}, k \geqslant 3$, связаны с тройками многочленов $H_{3 k-4}(x)$, $H_{3 k-2}(x)$ и $H_{3 k}(x)$.

Для получения этих следствий использовались равенства

$$
\begin{aligned}
& \Theta_{2,6}=\left(\frac{\theta_{3}}{3 !}\right)^{2}, \quad \Theta_{2,7}=2 \frac{\theta_{3}}{3 !} \frac{\theta_{4}}{4 !}, \quad \Theta_{2,8}=2 \frac{\theta_{3}}{3 !} \frac{\theta_{5}}{5 !}+\left(\frac{\theta_{4}}{4 !}\right)^{2} \\
& \Theta_{3,9}=\left(\frac{\theta_{3}}{3 !}\right)^{3}, \quad \Theta_{3,10}=3\left(\frac{\theta_{3}}{3 !}\right)^{2} \frac{\theta_{4}}{4 !}, \quad \Theta_{4,12}=\left(\frac{\theta_{3}}{3 !}\right)^{4} .
\end{aligned}
$$

В оценке остаточной части первого из следствий использовались явные выражения биномиальных коэффициентов, в оценке второго - явные выражения и неравенства $C_{n}^{k} \leqslant n^{k} / k$ !, в оценке остаточной части третьего - только такие неравенства.

Обратим внимание на то, что все слагаемые в оценке остаточной части разложения первого из следствий, убывающие при росте $n$ медленнее $1 / n^{2}$, «переходят» в главную часть разложения из второго следствия: величина $\beta_{4}\left(=\alpha_{4}\right)$ участвует в формировании $\theta_{4}$, а если во всех слагаемых оценки остаточной части первого разложения, убывающих как $1 / n^{3 / 2}$, снять знаки модуля, нормы, верхние индексы у величин $\theta_{4}^{(2)}$ и $\theta_{5}^{(3)}$ и заменить числа $B_{k, n}$ на функции $H_{k}(x) \varphi(x)$, то сумма этих слагаемых совпадет с суммой величин, убывающих как $1 / n^{3 / 2}$, в главной части разложения из второго следствия. Аналогичные слова можно сказать и о слагаемых, убывающих медленнее $1 / n^{5 / 2}$, в оценке остаточной части разложения из второго следствия. Отметим, что при четных $k$ справедливо равенство $\max _{-\infty<x<\infty}\left|H_{k}(x)\right| \varphi(x)=B_{k}$ и этот максимум достигается при $x=0$; указанный максимум не превосходит $B_{k}$ при всех натуральных $k$.

У читателя могут вызвать недоумение значения постоянных при некоторых комбинациях моментных характеристик в оценках остаточных частей разложений, точнее, в тех частях оценок величин $R$, которые при росте $n$ убывают с максимальной скоростью. Так, в оценке остаточной части третьего из приведенных следствий коэффициент при $\left(\left|\theta_{3}\right| / 3 !\right)\left(\left\|\theta_{7}^{(5)}\right\| / 7\right.$ !) (в сумме при $B_{10, n} / n^{3}$, она входит в оценку величины $r_{2}$ ) равен $1 / 2$ (это - оценка величины $(n-1) /(2 n)$ ). В связи с этим отметим, что в оценку остаточной части разложения для распределений с конечным седьмым моментом добавится слагаемое

$$
\frac{n-1}{2 n} \frac{\left|\theta_{7}^{(5)}\right|}{7 !} \frac{\left|\theta_{3}\right|}{3 !} \frac{B_{10, n}}{n^{3}}
$$

(оно войдет в оценку величины $R_{1}$ ), а для распределений с конечным 
восьмым моментом главная часть разложения содержит слагаемое

$$
\frac{1}{n^{3}} \frac{n-1}{n} \frac{\theta_{3}}{3 !} \frac{\theta_{7}}{7 !} H_{10}(x) \varphi(x) .
$$

Оказывается, что оценка теоремы при четных $m$ неулучшаема в том смысле, что если первое слагаемое в этой оценке (единственное, которое убывает как $n^{-m / 2}$ при росте $n$ ) умножить на любую постоянную $c<1$ (сколь угодно близкую к единице), то при некотором выборе $T>0$ для $x=0$ оценка станет неверной, начиная с некоторого $n$ (отметим, что неудачный выбор $T$ может привести к большим значениям чисел $B_{m+2, n}$ и сказанное может оказаться неверным). Для того чтобы убедиться в этом, покажем вначале, что для распределений с конечным четвертым моментом параметр $T$ можно выбрать так, что $B_{k, n} \rightarrow B_{k}$ при $n \rightarrow \infty$ для любого $k>0$.

Прежде всего заметим, что поскольку в определение функции $\mu(t)$ входит $e^{-t^{2} / 2}$, то $\liminf _{n \rightarrow \infty} B_{k, n} \geqslant B_{k}$ для любого $T>0$ и нам достаточно показать, что $\lim \sup _{n \rightarrow \infty} B_{k, n} \leqslant B_{k}$ при некотором $T>0$. Нам будет удобнее работать с верхним пределом величин

$$
\begin{aligned}
B_{k, n+1} & =\frac{1}{2 \pi} \int_{-T \sqrt{n+1}}^{T \sqrt{n+1}}|t|^{k} \mu^{n}\left(\frac{t}{\sqrt{n+1}}\right) d t \\
& =\frac{1}{2 \pi} \int_{-T \sqrt{n+1}}^{T \sqrt{n+1}}|t|^{k} \mu^{n}\left(\frac{t}{\sqrt{n}} \sqrt{\frac{n}{n+1}}\right) d t \\
& =\left(\sqrt{\frac{n+1}{n}}\right)^{(k+1) / 2} \frac{1}{2 \pi} \int_{-T \sqrt{n}}^{T \sqrt{n}}|t|^{k} \mu^{n}\left(\frac{t}{\sqrt{n}}\right) d t \\
& =\left(\sqrt{\frac{n+1}{n}}\right)^{(k+1) / 2} \bar{B}_{k, n+1} .
\end{aligned}
$$

Нетрудно проверить (см., например, $[1$, гл. 4, § 6]), что для распределений с $\theta_{4}<6$ справедлива оценка

$$
|f(t)| \leqslant \frac{1}{\sqrt{1+t^{2}}}
$$

при $|t| \leqslant \sqrt{2 \min \left(1,\left(6-\theta_{4}\right) /\left(6+\theta_{4}\right)\right)}$ и, кроме того, $e^{-t^{2} / 2} \leqslant 1 / \sqrt{1+t^{2}}$ при всех действительных $t$. Поэтому для распределений с $\theta_{4} \leqslant 3$ функция $\mu(t)$ не превосходит $1 / \sqrt{1+t^{2}}$ при $|t| \leqslant \sqrt{2 / 3}=T$ и для указанного $T$ и $n>k+1$

$$
\begin{gathered}
\bar{B}_{k, n+1}=\frac{1}{2 \pi} \int_{-T \sqrt{n}}^{T \sqrt{n}}|t|^{k} \frac{1}{\left(\sqrt{1+t^{2} / n}\right)^{n}} d t<\frac{1}{2 \pi} \int_{0}^{\infty} t^{k-1} \frac{1}{\left(1+t^{2} / n\right)^{n / 2}} d t^{2} \\
=\frac{n^{(k+1) / 2}}{2 \pi} \int_{0}^{\infty} u^{(k-1) / 2} \frac{1}{(1+u)^{n / 2}} d u=\frac{n^{(k+1) / 2}}{2 \pi} \mathrm{B}\left(\frac{k+1}{2}, \frac{n}{2}-\frac{k+1}{2}\right) \\
=\frac{2^{(k+1) / 2} \Gamma((k+1) / 2)}{2 \pi} \frac{(n / 2)^{(k+1) / 2} \Gamma(n / 2-(k+1) / 2)}{\Gamma(n / 2)} .
\end{gathered}
$$


Первая дробь в правой части последнего равенства совпадает с $B_{k}$, а вторая стремится к единице при $n \rightarrow \infty$.

Таким образом, для распределений $P$, у которых $\theta_{4} \leqslant 3$, выбор $T=$ $\sqrt{2 / 3}$ (можно брать любое $0<T \leqslant \sqrt{2 / 3}$ ) приводит к соотношению $B_{k, n} \rightarrow B_{k}$ при $n \rightarrow \infty$.

Пусть для распределения $P$ величина $\theta_{4}$ строго больше 3 . Так как $\theta_{4}\left(P_{l}\right)=\theta_{4}(P) / l$ для любого натурального $l$, то, взяв в качестве $l$ целую часть числа $\theta_{4}(P)$, мы получим распределение $Q=P_{l}$, для которого $\theta_{4}(Q) \leqslant 3 / 2$. Нетрудно проверить (переходя, например, к симметризованным случайным величинам), что для распределений $P$ с $\theta_{4}>0$ в некоторой окрестности нуля справедливо неравенство $|f(t / \sqrt{2})|^{2} \geqslant e^{-t^{2} / 2}$. Поэтому для распределения $P$ с $\theta_{4}>3$ для некоторого $\tau>0$ при $|t| \leqslant \tau$ функция $\mu(t)$ совпадает с $|f(t)|$. Введем функцию $N=N(n)$, равную целой части числа $n / l$, где выбор $l$ указан выше; ясно, что $n=l N+p$, где $0 \leqslant p<l$. Для любого $0<T \leqslant \tau$

$$
\begin{aligned}
\bar{B}_{k, n+1} & =\frac{1}{2 \pi} \int_{-T \sqrt{n}}^{T \sqrt{n}}|t|^{k}\left|f^{n}\left(\frac{t}{\sqrt{n}}\right)\right| d t \leqslant \frac{1}{2 \pi} \int_{-T \sqrt{n}}^{T \sqrt{n}}|t|^{k}\left|f^{l N}\left(\frac{t}{\sqrt{n}}\right)\right| d t \\
& =(\sqrt{n})^{k+1} \frac{1}{2 \pi} \int_{-T}^{T}|u|^{k}\left|f^{l N}(u)\right| d u \\
& =\left(\frac{n}{l N}\right)^{(k+1) / 2} \frac{1}{2 \pi} \int_{-T \sqrt{l N}}^{T \sqrt{l N}}|t|^{k}\left|f^{l N}\left(\frac{t}{\sqrt{l N}}\right)\right| d t \\
& =\left(1+\frac{p}{l N}\right)^{(k+1) / 2} \frac{1}{2 \pi} \int_{-T \sqrt{l} \sqrt{N}}^{T \sqrt{l} \sqrt{N}}|t|^{k}\left|q^{N}\left(\frac{t}{\sqrt{N}}\right)\right| d t,
\end{aligned}
$$

где $q-$ характеристическая функция распределения $Q$. Из сказанного выше следует, что

$$
|q(t)| \leqslant \frac{1}{\sqrt{1+t^{2}}} \quad \text { при }|t| \leqslant \sqrt{2 \min \left(1, \frac{6-\theta_{4}(Q)}{6+\theta_{4}(Q)}\right)} \leqslant 3 \sqrt{\frac{2}{15}}
$$

и если $T \sqrt{l} \leqslant 3 \sqrt{2 / 15}$, то для последнего интеграла справедлива та же оценка, что для интеграла из правой части первого равенства в цепочке (13) с заменой $n$ на $N$.

Отсюда следует, что для распределений $P$, у которых $\theta_{4}>3$, выбор $T=\min (\tau, 3 \sqrt{2 /(15 l)})$ вновь приводит к соотношению $B_{k, n} \rightarrow B_{k}$ при $n \rightarrow \infty$.

Можно проверить, что если при формировании главной части разложения мы будем использовать момент $\alpha_{m+2}\left(=\beta_{m+2}\right.$ для четных $\left.m\right)$, т.е. будем использовать величину

$$
\frac{\theta_{m+2}}{(m+2) !}=\frac{\alpha_{m+2}}{(m+2) !}+\frac{\theta_{m+2}^{(m)}}{(m+2) !}
$$


вместо $\theta_{m+2}^{(m)} /(m+2)$ !, то так построенное разложение будет аппроксимировать плотность $p_{n}(x)$ с точностью $o\left(n^{-m / 2}\right), n \rightarrow \infty$. Другими словами, для любого действительного $x$

$$
p_{n}(x)-\left(A_{n}(x)+\frac{\alpha_{m+2}}{(m+2) ! n^{m / 2}} H_{m+2}(x) \varphi(x)\right)=o\left(n^{-m / 2}\right), \quad n \rightarrow \infty,
$$

где $A_{n}(x)$ означает главную часть разложения из утверждения теоремы. Таким образом, для $x=0$ при четном $m$

$$
\left|p_{n}(0)-A_{n}(0)\right|=\frac{\beta_{m+2}}{(m+2) ! n^{m / 2}} B_{m+2}+o\left(n^{-m / 2}\right), \quad n \rightarrow \infty .
$$

Здесь мы использовали то, что для четного $m$ абсолютная величина свободного члена в многочлене $H_{m+2}(x)$ совпадает с моментом порядка $m+2$ стандартного нормального закона и $\varphi(0)=1 / \sqrt{2 \pi}$.

Отсюда и из только что доказанного утверждения о поведении величин $B_{k, n}$ и следует сформулированное выше утверждение о неулучшаемости оценки теоремы.

\section{СПИСОК ЛИТЕРАТУРЫ}

1. Сенатов В. В. Центральная предельная теорема: Точность аппроксимации и асимптотические разложения. М.: Книжный дом «Либроком», 2009, 352 с.

2. Сенатов B. B., Соболев B.H. О новых формах асимптотических разложений в центральной предельной теореме. - Теория вероятн. и ее примен., 2012, т. 57, в. 1 , с. $124-140$.

Поступила в редакцию 15.XI.2011 